\title{
Multimessenger astrophysics: When gravitational waves meet high energy neutrinos
}

\author{
Irene Di Palma* \\ Max-Planck-Institut für Gravitationsphysik, AEI, Callinstrasse 38, Hannover, Germany
}

For the ANTARES Collaboration, the LIGO Scientific Collaboration and the Virgo Collaboration

\section{A R T I C L E I N F O}

Available online 22 November 2013

\section{Keywords:}

Multimessenger analysis

Gravitational waves

High energy neutrinos

LIGO

Virgo

ANTARES

\begin{abstract}
A B S T R A C T
With recent development of experimental techniques that have opened new windows of observation of the cosmic radiation in all its components, multi-messenger astronomy is entering an exciting era. Many astrophysical sources and cataclysmic cosmic events with burst activity can be plausible sources of concomitant gravitational waves (GWs) and high-energy neutrinos (HENs). Such messengers could reveal hidden and new sources that are not observed by conventional photon astronomy, in particular at high energy. Requiring consistency between GW and HEN detection channels enables new searches and a detection would yield significant additional information about the common source. We present the results of the first search for gravitational wave bursts associated with high energy neutrino triggers, detected by the underwater neutrino telescope ANTARES in its 5 line configuration, during the fifth LIGO science run and first Virgo science run. No evidence for coincident events was found. We place a lower limit on the distance to GW sources associated with every HEN trigger. We are able to rule out the existence of coalescing binary neutron star systems and black hole-neutron star systems up to distances that are typically $5 \mathrm{Mpc}$ and $10 \mathrm{Mpc}$ respectively.
\end{abstract}

(c) 2013 Elsevier B.V. All rights reserved.

\section{The importance of multi-messenger astrophysics: feasible sources}

Many of the cataclysmic phenomena observed in our Universe are potential emitters of gravitational waves and high energy neutrinos. As cosmic messengers, both gravitational waves and neutrinos are complementary to photons in that they can escape very dense media and travel unabsorbed over cosmological distances, carrying information from the innermost regions of their astrophysical sources $[26,15]$. Hence, these messengers could also reveal new, hidden engines that have not yet been observed by conventional astronomy. Several known astrophysical sources are expected to produce both gravitational waves and high-energy neutrinos. Soft Gamma Repeaters are X-ray pulsars in our galaxy that exhibit bursts of soft $\gamma$ rays (flares), which may be associated with star-quakes [10]. The deformation of the star during the outburst could produce gravitational waves, while neutrinos could emerge from the flares. On the extragalactic scale, the most promising sources are $\gamma$ ray bursts (GRBs), which are known to be very energetic. The most popular models for GRBs involve either the

\footnotetext{
*Corresponding author. Tel.: + 4951176217153.

E-mail address: Irene.DiPalma@aei.mpg.de
}

collapse of a rapidly rotating massive star or the merger of a binary system of compact objects (neutron star/neutron star or black hole/ neutron star). In both scenarios, jets moving close to the speed of light are produced that give rise to the observed gamma-ray burst. The presence of protons or other hadrons in the jets would ensure the production of high-energy neutrinos, while gravitational waves would be produced by the binary merger or by any of several plausible mechanisms in the collapsing star scenario $[8,19,18]$.

\section{The analysis}

We present the results of the search for gravitational waves associated with 216 neutrino candidates that were identified by the underwater neutrino telescope ANTARES during the fifth LIGO science run and the first Virgo science run. The HEN data used in this analysis were collected from 2007 January 27 to 2007 September 30, for more details see Ref. [5]. The search uses the network analysis method described in the next section. The simplest search that may be performed combining GW and HEN data is a GW search around the neutrino arrival time and aimed at the location in the sky that the neutrino candidate is estimated to come from. We use a coherent search technique, called X-Pipeline [23], that has been utilized to perform searches for GWs in association with GRBs [3]. 
We adapt the method and the software to the specific GW-HEN problem and characterize its performance. X-Pipeline is a software package designed to target Gravitational-Wave Bursts (GWB) associated with external astrophysical triggers such as gamma-ray bursts or neutrinos. It performs a coherent analysis of the data from arbitrary networks of gravitational wave detectors, and it is robust against noise-induced glitches. This robustness is achieved by optimizing the search sensitivity based on the noise characteristics and the detector performance at the time of the trigger.

The first requirement imposed in a triggered search is that the GW candidate signal be coincident in time, within an astrophysically motivated window with the external trigger, the neutrino one in this case. By using a subset of the available GW data, a triggered search can be performed with a lower detection threshold than an un-triggered search, giving a higher detection probability at a fixed false alarm probability and better limits in the absence of detection. The number of accidental coincidences between GW detectors decreases with the size of the searched parameter space. Knowledge of the source direction allows us to search only a small part of the sky and veto candidate events seen in multiple detectors at times not consistent with the expected GW arrival time difference. Knowing the arrival time of the neutrino, we can restrict the time of the analysis to the specific search window and look for GW signals in coincidence with neutrino ones.

\section{Network of detectors}

Gravitational waves, predicted by Albert Einstein in 1916, are ripples in the space-time metric which are believed to propagate as a wave at the speed of light. These waves warp spacetime, changing the distance between nearby points in a characteristic pattern. Scientists attempt to detect gravitational waves using instruments called Michelson interferometers that bounce laser beams along two perpendicular arms. Measuring the interference between the beams allows to sense tiny variations in the arm lengths that may be caused by gravitational waves. LIGO is a network of three such instruments in the USA [2]: one in Livingston, LA (4 km arm length) and two in Hanford, WA (4 km and $2 \mathrm{~km}$ arm lengths in 2007). Virgo is a $3 \mathrm{~km}$ detector located at the European Gravitational Observatory in Cascina, Italy [4].

Neutrinos, on the other hand, are common yet enigmatic particles. They are stable, almost massless, and carry no electric charge, interacting with other particles through the weak force. The ANTARES collaboration has built an underwater neutrino telescope at a depth of $2475 \mathrm{~m}$ in the Mediterranean Sea to detect high-energy cosmic neutrinos using a three-dimensional array of roughly 900 light detectors (photomultipliers) distributed along 12 lines [9]. Unlike conventional telescopes, ANTARES looks downward, using the Earth to act as a shield, or filter, against all particles except neutrinos (which can easily pass through the Earth). A small fraction of the neutrinos passing upwards through the Earth will interact with the rock in the seabed to produce charged particles called muons, moving at near the speed of light. As these muons move through the water, they produce a flash of light called Cherenkov radiation. The photomultipliers detect this radiation, and from its arrival times the flight direction of the original neutrino can be estimated.

The data collected between February 9 and September 30, 2007, during the fifth LIGO science run and first Virgo science run and the 5 line configuration run of ANTARES, was used for a first joint search of GWs and HENs. In the next section, we report on the analysis of this data set.

\section{Search procedure: low- and high-frequency analyses}

Given our knowledge of possible GW sources, the most likely detectable signals at extra-galactic distances are in the lowfrequency band $(f \lesssim 500 \mathrm{~Hz}$ ), where our detectors have maximum sensitivity. At the same time, the computational cost of the search increases at high frequencies. This is in part not only due to the extra data to be analyzed because the frequency band is four times larger than the low frequency one, but also due to the need for finer-resolution sky grids to keep time delay errors much smaller than one GW period. We therefore split the gravitational wave band into two regions: $60-500 \mathrm{~Hz}$ and $500-2000 \mathrm{~Hz}$.

The low-frequency band is analyzed for all HEN triggers - such a search is computationally feasible while covering the highestsensitivity region of the GW detectors. However, compact objects such as neutron stars or collapsar cores have characteristic frequencies for GW emission above $500 \mathrm{~Hz}$. Such emissions might be detectable from galactic sources such as soft gamma repeater giant flares, or possibly at nearby galaxies. Since the computational cost of a high-frequency search for all HEN triggers is prohibitive with the current analysis pipeline, we perform the $500-2000 \mathrm{~Hz}$ analysis on the 3-line HEN triggers only. The 3-line events are a small subset $(\sim 10 \%)$ of the total trigger list and the most reliable, and have the smallest sky position uncertainties, and therefore the smallest computational cost for processing.

To reduce the computational cost further, we use the same sky grid for the high-frequency search as was used at low frequencies, after determining that the loss of sensitivity is acceptable. The high-frequency analysis is performed independent of the lowfrequency analysis (independent tuning, background estimation, etc.) using the same automated procedure. In the following sections we will present the results of the low-frequency and high-frequency searches separately.

\subsection{Handling 2-line ANTARES triggers}

The ANTARES Collaboration provided a list of 216 independent events as triggers for this analysis. Of these samples 198 were reconstructed with two lines and 18 were reconstructed with three lines. For a particle trajectory reconstructed from a Cherenkov cone giving hits on only two straight detector lines, there always exists an alternative trajectory having an identical $\chi^{2}$ value, but a different direction. The degenerate trajectory is the mirror image of the original track in the plane formed by the two lines [6]. The two trajectories will then have the same elevations relative to the detector zenith, but will differ in their azimuthal orientation. As a consequence, each event reconstructed with only two lines will have two equiprobable arrival directions, which must be taken into account during the GW analysis. Fig. 1 is a sky map of the full set of candidate HEN events showing the degenerate solutions connected with a black line and triggers reconstructed with three lines are in green. The estimated locations and their uncertainties may give rise to sky regions to be searched that are overlapping, as shown in Fig. 2. In our search we set up a grid that covers the error circle regions associated with each neutrino location. The GW search covers the sky position error box(es) with a set of points corresponding to different arrival time delays in the GW detectors, and each of these points is searched for separately. Each point is assigned a prior probability of how likely it is to be the true position of the source (HEN). Not all sky positions are equally probable to host the HEN source: a lognormal distribution [7] for the distance from the center of the error box describes the expected probability density for the neutrino. Such distribution is used to generate fake neutrino/GW triggers to feed in Monte-Carlo simulations aimed at measuring the detection efficiency of the pipeline. Fig. 3 shows an example of the HEN 


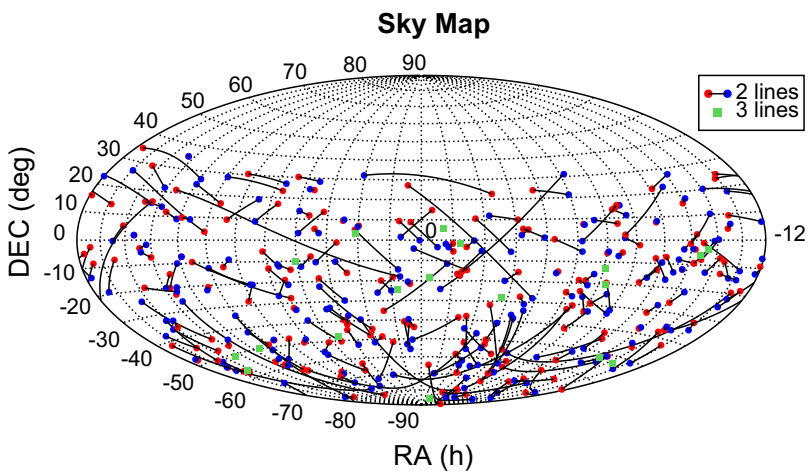

Fig. 1. Skymap of the full set of selected 216 HEN events in equatorial coordinates. A line connects the associated mirror solutions for events reconstructed with two lines. The squares indicate neutrinos reconstructed with three lines.

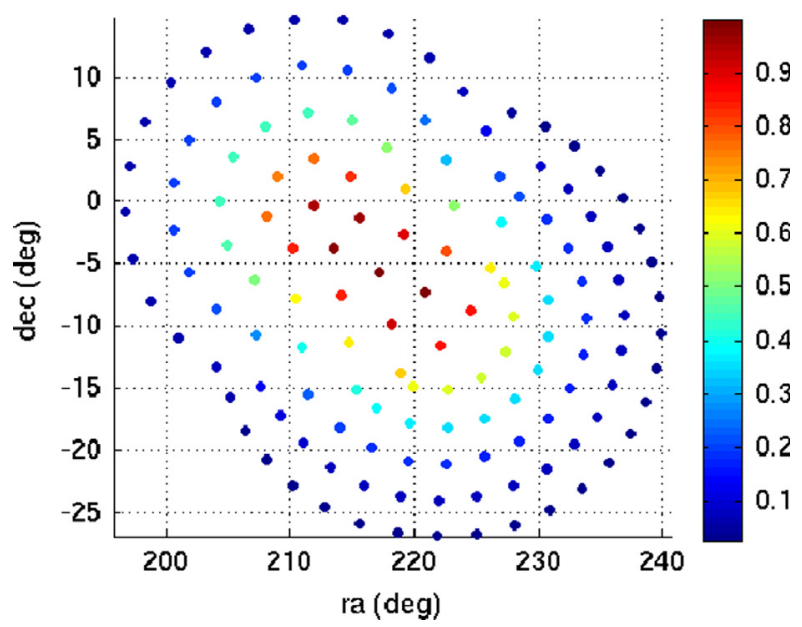

Fig. 2. Searching for one point and its mirror image at once, where the color bar shows the probability distribution. (For interpretation of the references to color in this figure caption, the reader is referred to the web version of this paper.)

probability distribution as a function of opening angle for a single HEN error region.

\subsection{The 2007 data search}

An unmodelled gravitational-wave search triggered by neutrinos is characterized by the neutrino sky position, the time of onset of neutrino emission, the trigger time $t_{0}$, and by the range of possible time delays, positive and negative, between the neutrino emission and the associated gravitational-wave emission. The time interval which is searched for GW candidate signals is called onsource. This search window is conservative enough to encompass most theoretical models of putative coincident GW-HEN emission. We consider a symmetric search window of $t_{0} \pm 500 \mathrm{~s}$ [11] even though the effective time in which we search for coincidences is $\left[t_{0}-496 \mathrm{~s}, t_{0}+496 \mathrm{~s}\right]$, due to $4 \mathrm{~s}$ of data that are thrown away from the beginning and end of each block due to filter transients.

Once the search is carried out, in order to evaluate the significance of the results one needs to estimate the background distributions. The off-source window is within $\pm 1.5 \mathrm{~h}$ of the neutrino time, excluding the on-source interval, see Fig. 4. In this way we assure that the background has similar statistical features as the data searched in association with the neutrino and at the same time does not contain any signal associated with the

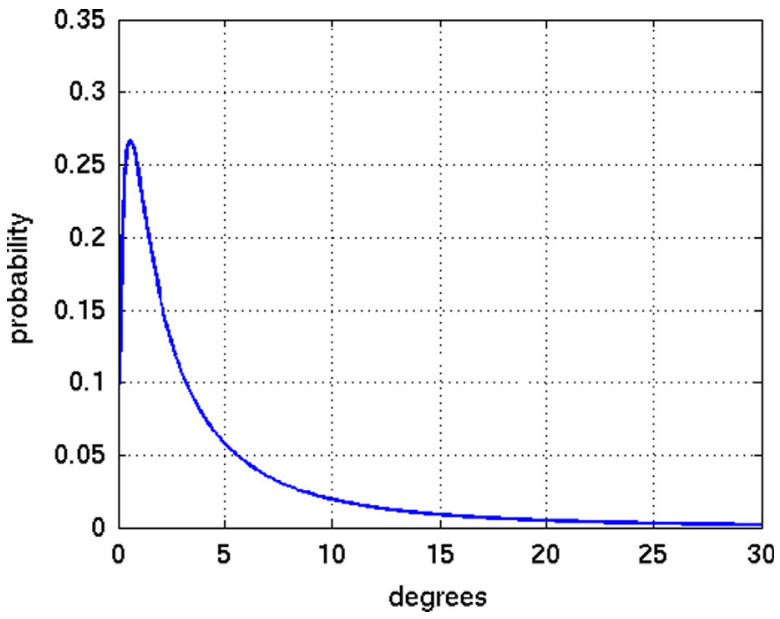

Fig. 3. The plot shows the lognormal probability distribution as a function of opening angle for one neutrino trigger error box.

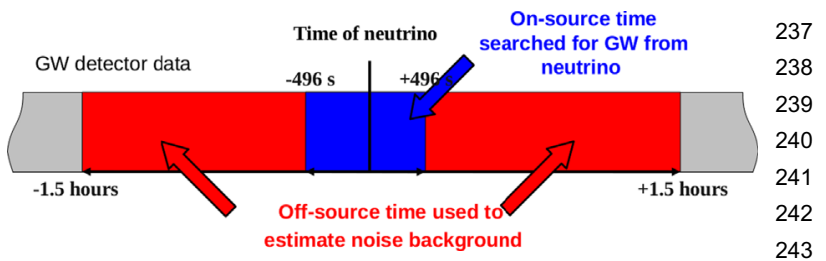

Fig. 4. This sketch shows the different between the on-source region and the offsource one. In the on-source region, that is the effective search window, we look for GW signals in coincidence with each neutrino trigger. The symmetric search window consists of $\pm 496 \mathrm{~s}$ around the neutrino time. The off-source window is defined as all data within $\pm 1.5 \mathrm{~h}$ of the neutrino time, excluding the on-source interval. This off-source data provides a sample of background that does not contain any signal associated with the neutrino event, but with statistical features similar to the data searched in association with the neutrino.

neutrino event. This time range is limited enough so that the detectors should be in a similar state of operation as during the neutrino on-source interval, but long enough to provide off-source segments for estimating the background.

From the full set of 216 independent neutrino candidates, 158 occurred at times when at least two gravitational-wave detectors were operating. Since two or more detectors are required to discriminate GW signals from background noise, in the following we consider only these remaining 158 HEN candidates: 144 2-line events and 14 3-line events. We analyze gravitational data in coincidence with 158 neutrino triggers for the low frequency search, and 14 neutrino triggers for the high frequency search. In the low frequency analysis, only one neutrino trigger had a corresponding GW event with false alarm probability below the threshold of $p=0.01$ to warrant further investigations, see Fig. 5 . We found no such candidates in the high frequency search.

This event came from analysis of the $\mathrm{H} 1, \mathrm{H} 2$, and V1 data. Follow-up checks were performed, including checks of detector performance recorded by monitoring programs and operator logs, and scans of data from detector and environmental monitoring equipment to look for anomalous behavior.

\subsubsection{Follow-up}

Further investigations are needed when one has a GW candidate event to establish if it is a real signal or a background event. Through this process, known as Follow-up, we verify the status of all detectors available at that time and check the monitors in the control room to provide information regarding loss of lock site disturbances, data corruptions and hardware injections. 


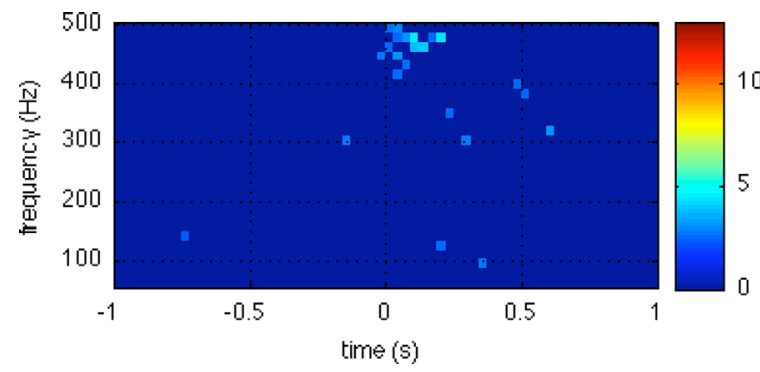

Fig. 5. Time-frequency map of our outlier for the low-frequency analysis. The color-bar shows the signal to noise ratio (SNR) of this event. (For interpretation of the references to color in this figure caption, the reader is referred to the web version of this paper.)

While these checks did not uncover a physical cause for the event, they did reveal that it occurred during a glitching period in V1. We conclude that we have no clear gravitational wave burst signal associated with any of our sample of 158 neutrino events.

\section{Search for a cumulative excess: binomial test}

A quantitative analysis of the significance of any candidate gravitational-wave event must take into account the trials factor due to the number of neutrino triggers analyzed. To do this we use the binomial test. Under the null hypothesis, the false alarm probabilities $p$ associated with the most significant GW candidate from every HEN search are expected to be uniformly distributed between 0 and 1 . The binomial test compares the measured $p$ values to the uniform distribution to determine if there is a statistically significant excess of small $p$ values (one or more) which may indicate the presence of gravitational wave signal.

Briefly, the binomial test sorts the set of $N$ measured loudest event probabilities in ascending order: $p_{1} \leq p_{2} \leq p_{3}, \ldots, p_{N}$. For each $i \in\left[1, N_{\text {tail }}\right]$ we compute the binomial probability $P_{\geq i}\left(p_{i}\right)$ of getting $i$ or more events with $p$ values $\leq p_{i}$ :

$P_{\geq i}\left(p_{i}\right)=\sum_{k=i}^{N} \frac{N !}{(N-k) ! k !} p_{i}^{k}\left(1-p_{i}\right)^{N-k}$

Here $N$ is the number of HEN analyzed ( 158 in the $60-500 \mathrm{~Hz}$ band and 14 in the $500-2000 \mathrm{~Hz}$ band). We only perform this test on $N_{\text {tail }}$, the top 5\% of analyzed HENs. Hence, $N_{\text {tail }}=8$ for the low frequency band and $N_{\text {tail }}=1$ for the high frequency band.

The lowest $P_{\geq i}\left(p_{i}\right)$ for $i \in\left[1, N_{\text {tail }}\right]$ is taken as the most significant deviation from the null hypothesis. To assess the significance of the deviation, we repeat the test using $p$ values drawn from a uniform distribution and count the fraction of such trials which give a lowest $P_{\geq i}\left(p_{i}\right)$ smaller than that computed from the true measured $p$ values.

Fig. 6 shows the cumulative distribution of $p$ values measured in the low- and high-frequency analyses. For the low-frequency search, bottom plot, the most significant deviation from the null hypothesis occurs for the third loudest event with $p_{3} \sim 0.013$. In the high-frequency analysis the largest deviation from the uniform distribution is constrained to happen for $p_{1}$ because we tested $N_{\text {tail }}=1$. In both cases the measured $p$ values are consistent with the null hypothesis.

\section{Upper limits and exclusion distances}

The sensitivity of the search of gravitational waves is determined by a Monte-Carlo analysis. For each HEN, we add (or 'inject') simulated gravitational wave burst signals into the detector data and repeat the analysis. We count an injected signal as 'detected' if it produces an event that is louder than the loudest on-source event within $100 \mathrm{~ms}$ of the injection time. For a given waveform morphology, we define the $90 \%$ confidence level upper limit on the signal amplitude as the minimum amplitude for which the detection probability is 0.9 or greater. From the upper limits on the GWB amplitude we derived the corresponding lower limits on the distance for each of the HENs analyzed. These limits are computed for circularly polarized $100 \mathrm{~Hz}$, $150 \mathrm{~Hz}$ and $300 \mathrm{~Hz}$ sine-Gaussian waveforms in the case of lowfrequency analysis and $554 \mathrm{~Hz}$ and $1000 \mathrm{~Hz}$ for the high frequency one. We compute the distance limits by assuming the source emitted $E_{G W}^{i s o}=0.01 M_{\odot} c^{2}=1.8 \times 10^{52}$ erg of energy isotropically in gravitational waves and use the following equation to infer a lower limit on $D$ :

$E_{G W}^{i s o} \approx \frac{\pi^{2} c^{3}}{G} D^{2} f_{0}^{2} h_{r s s}^{2}$

where $f_{0}$ is the central frequency, $h_{r s s}$ the root-sum-squared amplitude of the waveform and $D$ the distance of the source.

We can associate a physical distance to each amplitude for the sine-Gaussian waveforms as well, by assuming a fixed energy in gravitational waves. For concreteness, we select $E_{\mathrm{GW}}=10^{-2} M_{\odot} c^{2}$. This value corresponds to the optimistic limit of possible gravitationalwave emission by various processes in the collapsing cores of rapidly rotating massive stars $[13,22,14]$, more conservative estimates based on 3D simulations have been made in Refs. [12,20,24,21,25]. Our $90 \%$ confidence level lower limit on the distance to a GW source associated with a given HEN trigger is then the maximum distance $D_{90 \%}$ such that for any distance $D \leq D_{90 \%}$ there is a probability of at least 0.9 that such a GW signal would have produced an event louder than the loudest on-source event actually measured.

For each type of gravitational wave simulated, the distributions of exclusion distances for our neutrino sample are shown in Fig. 7. For binary neutron star systems of $(1.35-1.35) M_{\odot}$ and black holeneutron star systems of $(5-1.35) M_{\odot}$ typical distance limits are $5 \mathrm{Mpc}$ and $10 \mathrm{Mpc}$ respectively. For the sine-Gaussian waveforms in the low-frequency band the typical distance limits are between $5 \mathrm{Mpc}$ and $17 \mathrm{Mpc}$, while for those in the high-frequency band the typical limits are of order $1 \mathrm{Mpc}$, see Fig. 8.

\section{Conclusions}

The present analysis combines for the first time data from ANTARES, LIGO, and Virgo from 2007 to search for gravitational waves coincident with neutrinos. ANTARES data were used to determine the arrival time and direction of candidate high-energy neutrino events. The LIGO-Virgo data were then scanned for a gravitational wave around the time of each putative neutrino. By using a subset of the available GW data, the triggered search can be run with a lower event detection threshold, the false alarm rate for this search is thus about $\sim 100$ times lower than in an untriggered search [1], allowing lower thresholds with greater sensitivity to weak GWs.

At the same time by knowing the source direction we can search only a small part of the sky and veto candidate events seen in multiple detectors at times not consistent with the expected GW arrival time difference.

Hence, this leads to an improvement of a factor 1.5-2 in the maximum distance at which a GW source can be detected in comparison with GRBs triggered search [3] during the same period. This first joint GW-HEN search using 2007 data, obtained with the ANTARES HEN telescope and the Virgo/LIGO GW interferometers, opens the way to a novel multi-messenger astronomy. We place a lower limit on the distance to GW sources associated with every HEN trigger. We are able to rule out the existence of 

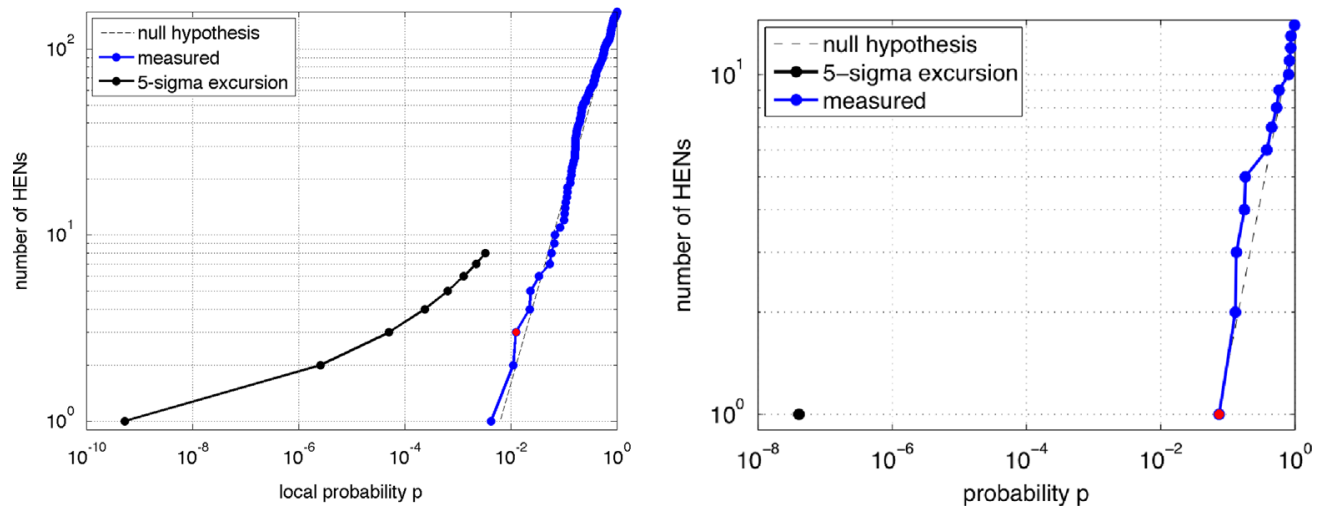

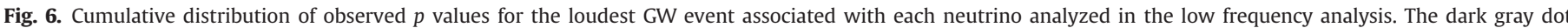

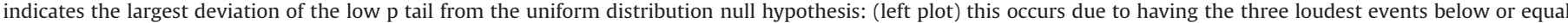

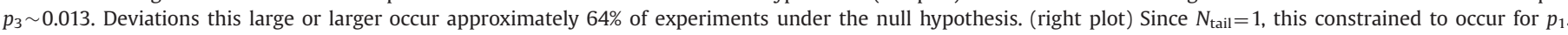

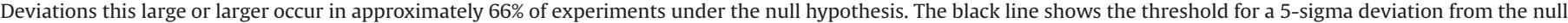
hypothesis.
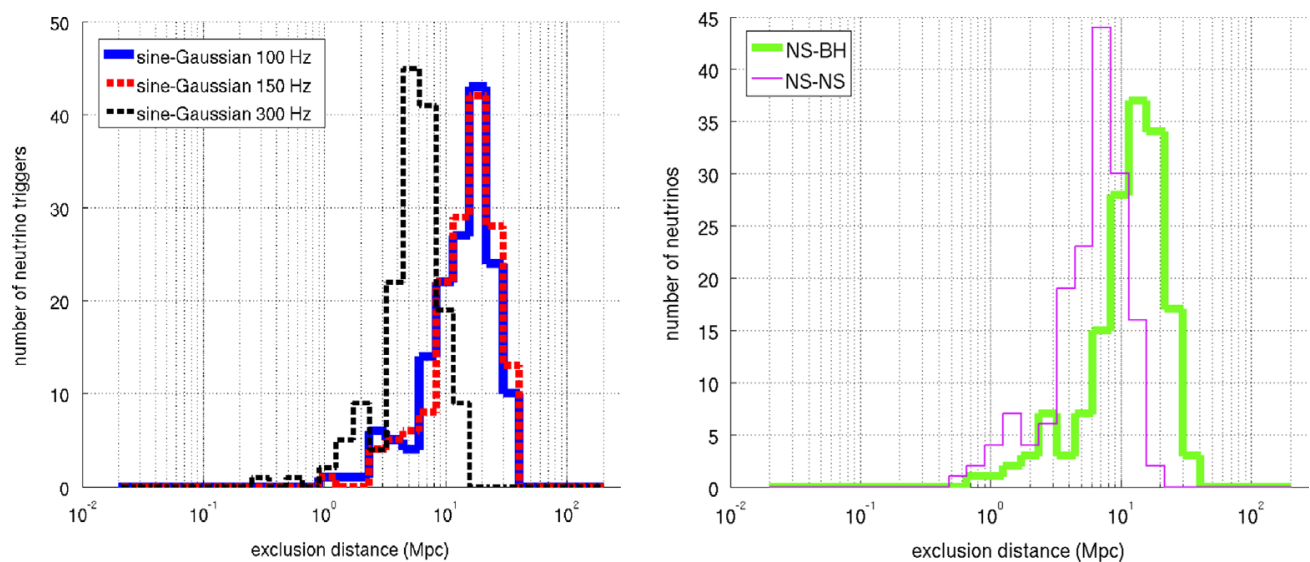

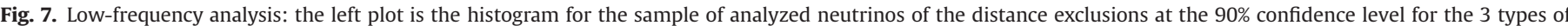

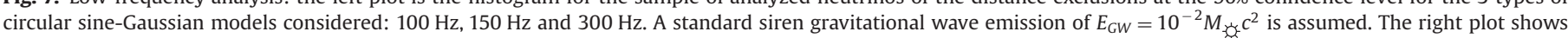

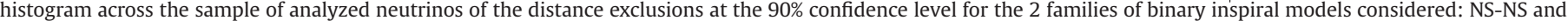
BH-NS.

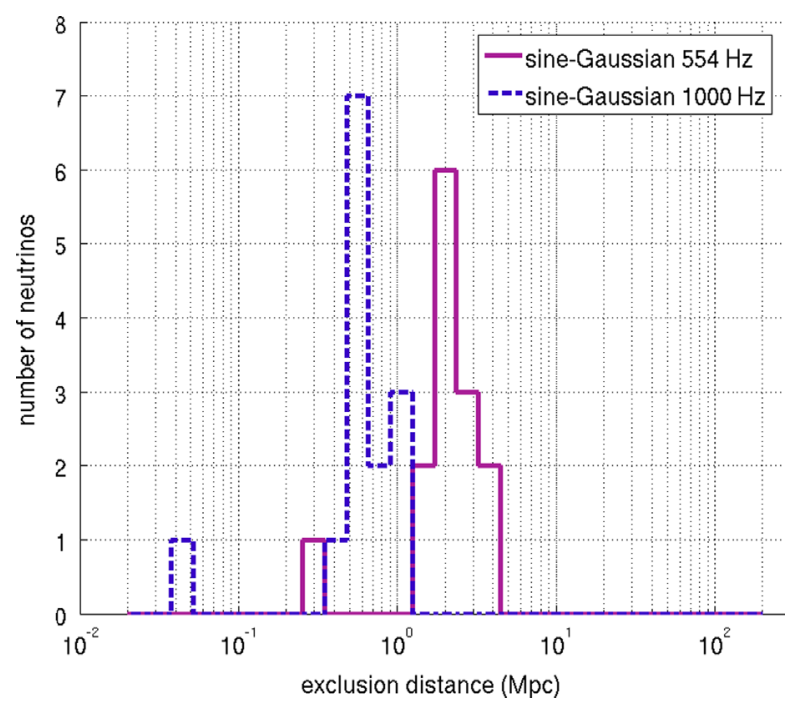

Fig. 8. High-frequency analysis: the histogram for the sample of analyzed neutrinos of the distance exclusions at the $90 \%$ confidence level for the 2 frequencies of circular sine-Gaussian models considered: $554 \mathrm{~Hz}$ and $1000 \mathrm{~Hz}$.

coalescing binary neutron star systems of $(1.35-1.35) M_{\odot}$ and black hole-neutron star systems of $(5-1.35) M_{\odot}$ up to distances that are typically of $5 \mathrm{Mpc}$ and $10 \mathrm{Mpc}$ respectively.
Similar analysis is in progress by using the sixth LIGO science run S6 and second and third Virgo science runs VSR2,3 covered the period from 7 July 2009 to 21 October 2010. Meanwhile, the ANTARES telescope has taken data with first 10 then 12 active lines since the end of December 2007. Another comparable effort is ongoing considering data from the LIGO/Virgo S5-VSR1 periods and the IceCube HEN telescope in its 22 string configuration. Future observing runs involving IceCube, KM3NeT [17], and the advanced LIGO and advanced Virgo projects [16] are likely to coincide as well. They will give other opportunities to look for potential coincident GW-HEN emissions.

\section{Acknowledgments}

This publication has been assigned LIGO Document Number LIGO-P1300097.

\section{References}

[1] J. Abadie, et al., Phys. Rev. D 81 (2010) 102001.

[2] B. Abbott, et al., Rep. Prog. Phys. 72 (2009) 076901 arXiv:0711:3041.

[3] B. Abbott, et al., Astrophys. J. 715 (2010) 1438 arXiv:0908.3824.

[4] F. Acernese, et al., Class. Quantum Gravity 25 (2008) 184001.

[5] Adriàn-Martínez, et al., J. Cosmol. Astropart. Phys. 1306 (2013) 008 arXiv:1205 3018.

[6] J.A. Aguilar, et al., Astropart. Phys. 34 (9) (2011) 652-662. 
[7] J. Aitchison, J. Brown, The Lognormal Distribution, Cambridge University Press, 1957.

[8] S. Ando, J. Beacom, Phys. Rev. Lett. 95 (6) (2005) 061103.

[9] E. Aslanides, et al., 2000, astro-ph/9907432.

[10] Y. Aso, et al., Class. Quantum Gravity 25 (2008) 114039.

[11] Baret et al., Bounding the Time Delay between High-energy Neutrinos and Gravitational-wave Transients from Gamma-ray Bursts. arXiv:1101.4669v1.

[12] H. Dimmelmeier, J.A. Font, E. Muller, Astrophys. J. 560 (2001) L163.

[13] C. Fryer, et al., Astrophys. J. 565 (2002) 430.

[14] C. Fryer, K. New, Living Rev. Relativ. 14 (2011).

[15] Guetta, et al., Astropart. Phys. 20 (2004) 429.

[16] G.M. Harry, et al., Class. Quantum Gravity 27 (2010) 084006
[17] J.J. Hernandez-Rey, J. Phys. Conf. Ser. 171 (2009) 012047.

[18] K. Ioka, et al., Astrophys. J. 633 (2005) 1013;

S. Kobayashi, P. Meszaros, Astrophys. J. 589 (2003) 861.

[19] P. Meszaros, E. Waxman, Phys. Rev. Lett. 87 (2001) 171102.

[20] C.D. Ott, Class. Quantum Gravity 26 (2009) 063001.

[21] C.D. Ott, et al., Phys. Rev. Lett. 106 (2011).

[22] A.L. Piro, E. Pfahl, Astrophys. J. 658 (2007) 1173.

[23] P. Sutton, et al., New J. Phys. 12 (2010) 053034 arXiv:0908.3665.

[24] S. Scheidegger, et al., Astron. Astrophys. 514 (2010).

[25] T. Takiwaki, K. Kotake, Astrophys. J. 743 (2011) 34.

[26] E. Waxman, J. Bahcall, Phys. Rev. Lett. 78 (1997) 2292. 